\title{
FATORES ASSOCIADOS À VIOLÊNCIA CONTRA AS MULHERES NA ATENÇÃO PRIMÁRIA DE SAÚDE
}

\section{Factors associated with violence against women in primary health care \\ Factores asociados con la violencia contra las mujeres en la atención primaria de salud}

\section{Eliane Rolim de Holanda}

Universidade Federal de Pernambuco - UFPE - Vitória de Santo Antão (PE) - Brasil

\section{Viviane Rolim de Holanda}

Universidade Federal de Pernambuco - UFPE - Vitória de Santo Antão (PE) - Brasil

\section{Marilena Silva de Vasconcelos}

Escola de Governo em Saúde Pública de Pernambuco - ESPPE - Recife (PE) - Brasil

\section{Valesca Patriota de Souza}

Universidade Federal de Pernambuco - UFPE - Vitória de Santo Antão (PE) - Brasil

\section{Marli Teresinha Gimeniz Galvão}

Universidade Federal do Ceará - UFC - Fortaleza (CE) - Brasil

\section{RESUMO}

Objetivo: Investigar os fatores associados à violência contra as mulheres na atenção primária à saúde baseando-se no Modelo de Atenção às Condições Crônicas (MACC). Métodos: Estudo retrospectivo e transversal, realizado em município da Zona da Mata Pernambucana, em que se analisaram 512 fichas de atendimento à mulheres em situação de violência, relativas ao período de 2008 a 2012, disponibilizadas pela Secretaria Executiva da Mulher. Para averiguar a relação entre as variáveis data da notificação, características sociodemográficas da vítima e do agressor (idade, estado civil, escolaridade, ocupação, renda, situação de moradia), tipo de violência sofrida, relação entre os envolvidos, tempo de relacionamento, causa atribuída à violência e uso de álcool e de droga, aplicou-se o teste Qui-Quadrado, com $p \leq 0,05$. Resultados: Verificou-se a predominância da violência física $(65 \%)$ praticada por companheiro conjugal $(p<0,001)$ em mulheres jovens $(p<0,001)$, com baixa escolaridade $(p<0,001)$, em união estável $(p<0,001)$ e com condições econômicas precárias $(p=0,013)$. O consumo de álcool pelo agressor $(\mathrm{p}<0,001)$ apareceu como principal comportamento de risco. Conclusão: A violência contra as mulheres obteve associação com a idade, com a escolaridade, com a renda da vítima, com o uso abusivo de álcool pelo agressor e com a relação conjugal.

Descritores: Violência Contra a Mulher; Atenção Primária à Saúde; Promoção da Saúde.

\section{ABSTRACT}

Objective: To assess the factors associated with violence against women in primary health care based on the Chronic Illness Care Model (Modelo de Atenção às Condições Crônicas - MACC). Methods: Retrospective cross-sectional study conducted in a municipality of the Zona da Mata in Pernambuco with medical records of 512 women subjected to violence from 2008 to 2012 made available by the Women's Executive Secretariat. The Chi-square test was used with a significance level of $p \leq 0.05)$ to determine the relationship between reporting data, sociodemographic characteristics of the victim and perpetrator (age, marital status, level of education, occupation, income, housing), type of violence, perpetrator-victim relationship, length of relationship, cause of the violence and use of alcohol and drugs. Results: There was a predominance of physical violence $(65 \%)$ perpetrated by marital partners $(p<0.001)$ against young women $(p<0.001)$ with low levels of education $(p<0.001)$ in a common-law marriage $(p<0.001)$ and poor economic conditions $(p=0.013)$. Alcohol use by perpetrators $(p<0.001)$ was the main risk behavior. Conclusion: Violence against women was associated with the victims' age, level of education, income, alcohol abuse by perpetrators, and marital relationships.

Descriptors: Violence Against Women; Primary Health Care; Health Promotion. 


\section{RESUMEN}

Objetivo: Investigar los factores asociados con la violencia contra las mujeres en la atención primaria de salud basado en el Modelo de Atención de las Condiciones Crónicas (MACC). Métodos: Estudio retrospectivo y transversal realizado en un municipio de la Zona de la Mata de Pernambuco en el cual se analizaron 512 fichas de asistencia a las mujeres en situación de violencia relativas al periodo entre 2008 y 2012 disponibles en la Secretaria Ejecutiva de la Mujer. Para verificar la relación entre las variables fecha de notificación, características sociodemográficas de la víctima y del agresor (edad, estado civil, escolaridad, ocupación, renta, situación de vivienda), tipo de violencia sufrida, relación entre los involucrados, tiempo de relacionamiento, causa de la violencia y uso de alcohol y droga se aplicó la prueba Chi-Cuadrado con el $p \leq 0,05$. Resultados: Se verificó la predominancia de la violencia fisica (65\%) practicada por el compañero conyugal $(p<0,001)$ en mujeres jóvenes $(p<0,001)$, de baja escolaridad $(p<0,001)$, en unión estable $(p<0,001)$ y con condiciones económicas precarias $(p=0,013)$. El consumo de alcohol por el agresor ( $<<0,001)$ fue la principal conducta de riesgo. Conclusión: La violencia contra las mujeres estuvo asociada con la edad, la escolaridad, la renta de la víctima, el uso abusivo del alcohol por el agresor y la relación conyugal.

Descriptores: Violencia contra la Mujer; Atención Primaria de Salud; Promoción de la Salud.

\section{INTRODUÇÃO}

A violência contra as mulheres ocorre frequentemente em diversas culturas e cenários, sendo amplamente reconhecida como grave problema de saúde pública. Reproduz as relações de poder entre os homens e as mulheres na sociedade e representa uma das principais formas de violação dos direitos humanos. Pode ser definida como qualquer ação ou omissão fundamentada no gênero que cause morte, lesão, sofrimento físico, sexual ou psicológico e dano moral ou patrimonial à mulher ${ }^{(1)}$.

No Brasil, a Política Nacional de Atenção Integral à Saúde da Mulher (PNAISM) declarou dentre os seus objetivos a incorporação da integralidade na perspectiva de gênero para alcançar a promoção e a humanização da saúde como princípios norteadores ${ }^{(2)}$. Como pauta de tal política, priorizou-se a organização de redes integradas para o empoderamento feminino em situação de violência doméstica e sexual e a implementação de políticas de promoção da saúde ${ }^{(3)}$.

As redes de atenção à saúde devem funcionar intersetorialmente, articulando serviços de saúde do nível primário com os demais níveis por meio de ações vinculadas entre si para ofertar assistência contínua, de qualidade, humanizada, equânime e segura à população. Tais redes funcionam a partir de elementos constitutivos, sendo eles: a população, a estrutura operacional e os modelos de atenção à saúde para as condições agudas ou para as crônicas ${ }^{(4,5)}$.

A função dos modelos de atenção à saúde é organizar o funcionamento das redes, articulando as relações entre os seus componentes e as intervenções sanitárias, definidas a partir do conceito prevalecente de saúde, das situações demográfica e epidemiológica, e dos determinantes sociais da saúde vigentes em um certo tempo e sociedade ${ }^{(4)}$.

Nesse contexto, aponta-se a proposta do Modelo de Atenção às Condições Crônicas (MACC) como uma alternativa viável para o enfretamento da violência contra a mulher sob um prisma mais abrangente. A fundamentação conceitual do MACC adapta-se às exigências do sistema público de saúde brasileiro. Trata-se da junção de três outros modelos de atenção à saúde, a saber: o modelo de atenção crônica (CCM), o modelo de pirâmide de risco e o modelo da determinação social da saúde, subdividindo-se em cinco níveis de atuação de acordo com as populações estratificadas pelo modelo(5)

O nível 1 relaciona-se às intervenções de promoção da saúde e tem foco nos determinantes sociais intermediários ligados às condições de vida e de trabalho da população. O nível 2 desenvolve intervenções de prevenção das condições de saúde, considerando o autocuidado apoiado e os determinantes proximais em subpopulações com fatores de risco associados aos comportamentos e aos estilos de vida. Nesses dois primeiros níveis, as equipes de saúde atuam antes que condições crônicas se estabeleçam. A partir do nível 3 são identificadas as alterações da saúde. Os níveis 3, 4 e 5 do MACC estão vinculados aos indivíduos com condição crônica complexa instalada e requerem intervenções predominantemente clínicas ${ }^{(5)}$.

A violência tem se configurado como uma condição crônica, pois as suas interfaces vêm causando repercussões graves que resultam em sofrimento físico e/ou psíquico, incluso nesse aspecto a depressão, o estresse pós-traumático, a tendência ao suicídio e o consumo de substâncias lícitas e ilícitas. É fator de risco para o desenvolvimento de diversos problemas de saúde, gerando altos custos sobre a produção econômica ${ }^{(6-8)}$.

É imprescindível dimensionar com mais precisão a ocorrência desse fenômeno, dada a sua complexidade e a gênese multicausal, por meio da realização de pesquisas que ampliem a visibilidade do tema. Assim, a questão norteadora do presente estudo é: Qual a prevalência dos fatores associados à violência contra mulheres usuárias dos serviços de atenção primária à saúde? Este estudo constitui importante contribuição para a área da saúde, uma vez que fornece subsídios para se repensar o modo de cuidar da mulher em situação de violência a partir do MACC.

Diante do exposto, objetivou-se investigar os fatores associados à violência contra as mulheres na atenção primária de saúde baseando-se no MACC. 


\section{MÉTODOS}

Trata-se de estudo retrospectivo, transversal, realizado no município de Vitória de Santo Antão, Pernambuco, Brasil, localizado na Zona da Mata Sul de Pernambuco, região Nordeste do Brasil.

Realizou-se amostragem do tipo censitária, composta por toda a população dos casos atendidos de violência contra a mulher, no período de junho de 2008 a dezembro de 2012, na Secretaria Especial da Mulher. Essa secretaria era um órgão vinculado à Prefeitura (https://mulheresdavitoria.wordpress.com/2008/03/), e, no referido período, se constituía como único lócus de atendimento à mulher vítima de violência no município, aspecto que justifica a publicação dos achados, dado a importância histórica para conhecimento da realidade local em uma região com forte legado patriarcal na divisão de gênero e carente de estudos relativos à violência doméstica por subnotificações. Somente a partir de 2013 o município recebeu a Delegacia da Mulher e o Centro de Referência Especializado no Atendimento à Mulher em Situação de Violência.

Coletaram-se os dados no período de janeiro a abril de 2013, em sala reservada, sob monitoramento de funcionários do serviço, considerando o sigilo que envolve atendimentos dessa natureza. Para a coleta, utilizaram-se fichas de elaboração própria do serviço para o atendimento das mulheres em situação de violência. As fichas continham as seguintes variáveis: data da notificação, características sociodemográficas da vítima e do agressor (idade, estado civil, escolaridade, ocupação, renda, situação de moradia), tipo de violência sofrida, relação entre os envolvidos, tempo de relacionamento, causa atribuída à violência, uso de álcool e de droga.

Importante referir que essa etapa aconteceu manualmente, ficha a ficha. Observou-se o preenchimento das fichas de atendimento com variáveis incompletas, entretanto, não se descartou nenhuma ficha. Assim sendo, o estudo abrangeu $100 \%$ dos atendimentos no período selecionado, totalizando 512 casos. Nos resultados apresentados, os valores das variáveis diferem do total de casos analisados devido às informações não preenchidas.

Construiu-se um banco de dados no programa Epi Info 2000, exportando-o posteriormente para o software SPSS, versão 20.0. Para a análise, calcularam-se as taxas de prevalência, as frequências percentuais e as distribuições de frequência das variáveis analisadas. Aplicou-se o teste Qui-quadrado para a comparação de proporção e, em seguida, analisou-se os fatores associados à violência e o perfil das vítimas com a adoção de significância estatística de 5\%.

A discussão dos resultados fundamentou-se nos pressupostos teóricos contidos nos níveis 1 e 2 do MACC $^{(5)}$, por entender que contemplam o manejo da violência de gênero como uma condição crônica na atenção primária e possuem enfoque na promoção da saúde.

O presente estudo considerou as diretrizes regulamentadoras da pesquisa envolvendo seres humanos e obteve aprovação pelo Comitê de Ética e Pesquisa da Universidade Federal de Pernambuco, segundo Protocolo no 495/11.

\section{RESULTADOS}

Dos 512 casos de mulheres analisados, quanto às características sociodemográficas, constatou-se a maior prevalência naquelas com idade entre 19 e 30 anos ( $<<0,001)$, em união estável $(p<0,001)$, com ensino fundamental $(p<0,001)$, moradoras da zuna urbana $(\mathrm{p}<0,001)$, com renda de menos de um salário mínimo $(\mathrm{p}=0,013)$ e residindo em casa própria $(\mathrm{p}<0,001)$, conforme consta na Tabela I. O teste de comparação de proporção apresentou-se significativo em todos os fatores avaliados.

Tabela I - Caracterização sociodemográfica das mulheres vítimas de violência. Vitória de Santo Antão, Pernambuco, 2013.

\begin{tabular}{lccc}
\hline Fator avaliado & $\mathbf{n}$ & $\mathbf{\%}$ & p-valor $^{\mathbf{1}}$ \\
\hline Idade (anos) *(n=439) & 45 & 10,3 & \\
<18 & 177 & 40,3 & \\
19 a 30 & 106 & 24,1 & $<0,001$ \\
11 a 40 & 71 & 16,2 & \\
41 a 50 & 25 & 5,7 & \\
> a 60 & 15 & 3,4 & \\
Estado civil *(n=231) & & & \\
Casada & 67 & 29,0 & \\
Divorciada/separada & 20 & 8,7 & \\
Solteira & 71 & 30,7 & \\
União estável & 73 & 31,6 & \\
Escolaridade *(n=139) & & & \\
Analfabeta & 14 & 10,1 & \\
Ensino fundamental & 69 & 49,6 & \\
Ensino médio & 44 & 31,7 & \\
Ensino superior & 12 & 8,6 & \\
& & & \\
\hline
\end{tabular}




\begin{tabular}{|c|c|c|c|}
\hline \multicolumn{4}{|c|}{ Zona $*(n=400)$} \\
\hline Rural & 27 & 6,7 & \multirow{2}{*}{$<0,001$} \\
\hline Urbana & 373 & 93,3 & \\
\hline \multicolumn{4}{|c|}{ Renda própria * $(\mathrm{n}=117)$} \\
\hline Sim & 72 & 61,5 & \multirow{2}{*}{0,013} \\
\hline Não & 45 & 38,5 & \\
\hline \multicolumn{4}{|c|}{ Situação de moradia * $(n=84)$} \\
\hline Própria & 38 & 45,2 & \multirow{4}{*}{$<0,001$} \\
\hline Alugada & 34 & 40,5 & \\
\hline Cedida & 3 & 3,6 & \\
\hline Outras & 9 & 10,7 & \\
\hline
\end{tabular}

${ }^{1}$ p-valor do teste Qui-quadrado para comparação de proporção. *Os totais diferem devido ao preenchimento nas fichas de atendimento com variáveis incompletas.

Com relação à prevalência do tipo de violência sofrida, verificou-se a violência física como a mais frequente na população do estudo $(65,0 \%, 333$ casos), seguida pela violência psicológica (60,4\%, 309 casos), violência moral ( $17 \%$, 87 casos), violência patrimonial $(10,4 \%, 53$ casos) e violência sexual $(6,1 \%, 31$ casos).

A Tabela II apresenta a prevalência da violência segundo o tipo e os fatores associados ao perfil da vítima. Observa-se que a violência física $(\mathrm{p}<0,001)$ e a sexual $(\mathrm{p}=0,002)$ ocorreram com maior frequência nas mulheres com idade menor de 18 anos, enquanto a violência psicológica $(\mathrm{p}<0,001)$ predominou nas mulheres com idade de 31 a 40 anos. As mulheres com idade maior que 60 anos sofreram mais violência moral $(p<0,001)$ e patrimonial $(p<0,001)$. Identificou-se significância no teste de comparação de proporção em todos os tipos de violência avaliados, indicando que a prevalência diferiu segundo as faixas etárias.

Quanto ao estado civil, as mulheres em união estável sofreram maior número de violência física ( $\mathrm{p}<0,001)$. As casadas passaram por mais violência psicológica $(p=0,002)$ e moral $(p<0,001)$. Em relação à escolaridade, as mulheres que estudaram até o ensino fundamental sofreram mais violência sexual $(\mathrm{p}=0,028)$ e aquelas com ensino médio, a violência física ( $\mathrm{p}<0,001)$. A maior prevalência da violência psicológica ocorreu em mulheres que possuíam nível superior $(\mathrm{p}<0,001)$.

A violência psicológica prevaleceu nas mulheres que residem na zona rural $(p<0,001)$ e os demais tipos de violência, nas mulheres da zona urbana. As mulheres sem renda própria apresentaram maior prevalência de violência física, sexual e patrimonial, e aquelas que possuíam renda, violência psicológica $(\mathrm{p}=0,010)$ e moral $(\mathrm{p}=0,041)$. Mesmo sendo observadas essas diferenças na prevalência dos tipos de violência entre as situações da renda própria, o teste de comparação de proporção apontou significância apenas na violência psicológica e moral indicando que a ocorrência nesses grupos avaliados diferiu estatisticamente. Quanto à situação de moradia, as mulheres com residência própria sofreram mais violência do tipo física $(\mathrm{p}<0,001)$.

Tabela II - Prevalência da ocorrência da violência segundo o tipo e os fatores associados da vítima. Vitória de Santo Antão, Pernambuco, 2013.

\begin{tabular}{lccccc}
\hline Fator avaliado & Física & Sexual & $\begin{array}{c}\text { Tipo de violência } \\
\text { Psicológica }\end{array}$ & Moral & Patrimonial \\
\hline Idade (anos) & & & & & \\
$<18$ & $35(77,8)$ & $9(20,0)$ & $19(42,2)$ & $4(8,9)$ & $1(2,2)$ \\
19 a 30 & $117(66,1)$ & $5(2,8)$ & $108(61,0)$ & $27(15,3)$ & $25(14,1)$ \\
31 a 40 & $66(62,3)$ & $12(11,3)$ & $76(71,7)$ & $22(20,8)$ & $18(17,0)$ \\
41 a 50 & $48(67,6)$ & $3(4,2)$ & $47(66,2)$ & $10(14,1)$ & $0(0,0)$ \\
51 a 60 & $14(56,0)$ & $1(4,0)$ & $17(68,0)$ & $5(20,0)$ & $1(4,0)$ \\
> 60 & $8(53,3)$ & $1(6,7)$ & $9(60,0)$ & $7(46,7)$ & $5(33,3)$ \\
p-valor ${ }^{1}$ & $<0,001$ & 0,002 & $<0,001$ & $<0,001$ & $<0,001$ \\
Estado civil & & & & \\
Casada & $40(59,7)$ & $7(10,4)$ & $47(71,1)$ & $18(26,9)$ & $7(10,4)$ \\
Divorciada/separada & $8(40,0)$ & $1(5,0)$ & $14(70,0)$ & $1(5,0)$ & $3(15,0)$ \\
Solteira & $41(57,7)$ & $7(9,9)$ & $48(67,6)$ & $15(21,1)$ & $7(9,9)$ \\
União estável & $58(79,5)$ & $8(11,0)$ & $51(69,9)$ & $10(13,7)$ & $7(9,6)$ \\
p-valor ${ }^{1}$ & $<0,001$ & 0,148 & $<0,001$ & 0,002 & 0,572 \\
Escolaridade & & & & \\
Analfabeta & $10(71,4)$ & $0(0,0)$ & $8(57,1)$ & $5(35,7)$ & $1(7,1)$ \\
Ensino fundamental & $49(71,0)$ & $12(17,4)$ & $55(79,7)$ & $9(13,0)$ & $6(8,7)$ \\
Ensino médio & $32(72,7)$ & $7(15,9)$ & $35(79,5)$ & $5(11,4)$ & $8(18,2)$ \\
Ensino superior & $6(50,0)$ & $2(16,7)$ & $11(91,7)$ & $3(25,0)$ & $2(16,7)$ \\
p-valor ${ }^{1}$ & $<0,001$ & 0,028 & $<0,001$ & 0,327 & 0,052 \\
\hline
\end{tabular}




\begin{tabular}{lccccc}
\hline Zona & & & & & \\
Rural & $15(55,6)$ & $1(3,7)$ & $19(70,4)$ & $4(14,8)$ & $2(7,4)$ \\
Urbana & $245(65,7)$ & $25(6,7)$ & $232(62,2)$ & $70(18,8)$ & $45(12,1)$ \\
p-valor & $<0,001$ & $<0,001$ & $<0,001$ & $<0,001$ & $<0,001$ \\
Renda própria & & & & & \\
Sim & $42(58,3)$ & $9(12,5)$ & $60(83,3)$ & $17(23,6)$ & $4(5,6)$ \\
Não & $32(71,1)$ & $10(22,2)$ & $35(77,8)$ & $7(15,6)$ & $7(15,6)$ \\
p-valor & 0,245 & 0,819 & 0,010 & 0,041 & 0,366 \\
Moradia & & & & & \\
Própria & $28(73,7)$ & $11(28,9)$ & $37(97,4)$ & $5(13,2)$ & $3(7,9)$ \\
Alugada & $19(55,9)$ & $7(20,6)$ & $30(88,2)$ & $4(11,8)$ & $4(11,8)$ \\
Cedida & $2(66,7)$ & $1(33,3)$ & $3(100,0)$ & $0(0,0)$ & $1(33,3)$ \\
Outras & $5(55,6)$ & $0(0,0)$ & $7(77,8)$ & $0(0,0)$ & $1(11,1)$ \\
p-valor & $<0,001$ & 0,018 & $<0,001$ & 0,739 & 0,392 \\
\hline
\end{tabular}

${ }^{1} \mathrm{p}$-valor do teste Qui-quadrado para comparação de proporção

A maior parte das mulheres indicou os companheiros conjugais como agressores $(53,4 \%, 198$ casos). As mulheres que possuíam mais de 10 anos de relacionamento $(37,2 \%, 54$ casos) passaram por mais episódios violentos do que aquelas que apresentavam tempo inferior. As mulheres referiram sofrer violência frequentemente $(67,9 \%, 76$ casos), atribuindo a agressão ao uso abusivo do álcool $(38,4 \%, 53$ casos). Elas apontaram que o agressor costumava fazer uso rotineiro de álcool e/ou outras drogas $(86,2 \%, 125$ casos). O teste de comparação de proporção revelou-se significativo em todos os fatores avaliados, indicando frequência nos casos de violência conforme consta na Tabela III.

Tabela III - Características do agressor. Vitória de Santo Antão, Pernambuco, 2013.

\begin{tabular}{|c|c|c|c|}
\hline Fator avaliado & $\mathbf{n}$ & $\%$ & p-valor \\
\hline \multicolumn{4}{|c|}{ Relação com o agressor *(n=371) } \\
\hline Parente de grau $1^{\circ}$ e $2^{\circ}$ & 47 & 12,7 & \multirow{4}{*}{$<0,001$} \\
\hline Companheiro conjugal & 198 & 53,4 & \\
\hline Ex-companheiro & 115 & 31,0 & \\
\hline Conhecido & 11 & 2,9 & \\
\hline \multicolumn{4}{|c|}{ Tempo de relacionamento $($ anos $) *(n=145)$} \\
\hline 1 a 4 & 51 & 35,2 & \multirow{4}{*}{$<0,001$} \\
\hline 5 a 7 & 24 & 16,6 & \\
\hline 8 a 10 & 16 & 11,0 & \\
\hline 10 ou mais & 54 & 37,2 & \\
\hline \multicolumn{4}{|l|}{ Frequência * $(n=112)$} \\
\hline Esporádica & 16 & 14,2 & \multirow{3}{*}{$<0,001$} \\
\hline Frequente & 76 & 67,9 & \\
\hline Rara & 20 & 17,9 & \\
\hline \multicolumn{4}{|c|}{ A que atribui a violência * $(n=138)$} \\
\hline Uso abusivo do álcool & 53 & 38,4 & \multirow{4}{*}{$<0,001$} \\
\hline Ciúmes & 43 & 31,2 & \\
\hline Temperamento agressivo & 31 & 22,5 & \\
\hline Outros & 11 & 7,9 & \\
\hline \multicolumn{4}{|c|}{ Uso de álcool/outras drogas * $(\mathrm{n}=145)$} \\
\hline $\operatorname{Sim}$ & 125 & 86,2 & \multirow{2}{*}{$<0,001$} \\
\hline Não & 20 & 13,8 & \\
\hline
\end{tabular}

${ }^{1}$ p-valor do teste Qui-quadrado para comparação de proporção; * Os totais diferem devido ao preenchimento nas fichas de atendimento com variáveis incompletas

\section{DISCUSSÃO}

A violência contra a mulher é um fenômeno complexo e multifatorial, pautado em questões de gênero e hierarquia de poder, relacionado à condição de desigualdade da mulher em relação ao homem, construída e naturalizada historicamente ${ }^{(9)}$. Ressalta-se, assim, a importância de se identificar os fatores associados à violência contra as mulheres, uma vez que aspectos sociodemográficos, comportamentais e culturais podem intensificar a violência praticada pelo parceiro.

Os achados da presente pesquisa revelam a predominância de vítimas com idade jovem, de baixa escolaridade, em união estável e com condições econômicas precárias, corroborando com outras pesquisas que atribuem essas características à maior vulnerabilidade das mulheres frente aos diversos tipos de violência ${ }^{(8,10-12)}$. 
Os resultados advindos da análise bivariada diferem de outros estudos, em que predominou a violência psicológica, seguida da física e sexual ${ }^{(6,11,13)}$. Os conflitos conjugais tende a se iniciar a partir de agressões verbais, evoluindo para violência física ${ }^{(6)}$, e o abuso psicológico é a violência mais negligenciada, sendo raramente reconhecida ${ }^{(13)}$.

A mulher possui maior vulnerabilidade frente a episódios de violência, independente do seu nível de escolaridade e condição socioeconômica ${ }^{(12)}$. No entanto, acrescenta-se que poucos anos de estudo e dependência financeira do companheiro conjugal intensificam a frequência de conflitos entre os casais, já que mulheres menos esclarecidas teriam maior dificuldade para romper os ciclos de agressões ${ }^{(6,14,15)}$. A melhor escolaridade parece estar associada a um empoderamento pessoal da mulher, promovendo redução da tolerância à violência ${ }^{(16)}$. Quanto mais a mulher se qualifica, mais chance tem de encontrar trabalho remunerado, melhorando, dessa forma, a autoestima e a independência ${ }^{(17)}$. Portanto, considera-se a necessidade de ações que permitam aos profissionais de saúde conceder condições de acolhimento e escuta, com vista à conscientização e ao empoderamento das mulheres vitimadas, para descontinuação de relações destrutivas.

O companheiro conjugal relacionou-se como o mais importante perpetrador de violência contra a mulher na presente pesquisa. Este achado é concordante com outras pesquisas sobre a temática ${ }^{(11,15)}$, que relacionam tal fato à desigualdade de direitos, que impõe à mulher a obediência e a submissão ao homem numa situação de inferioridade e dominação masculina.

$\mathrm{Na}$ maioria dos casos, a mulher opta por não informar a agressão sofrida pelo companheiro conjugal e muitos dos atendimentos decorrentes dessa violência não são identificados nos serviços de saúde ${ }^{(18)}$. Em virtude disso, estudos ${ }^{(16,19-21)}$ destacaram que os profissionais de saúde, principalmente da atenção primária de saúde, enfrentam dificuldades para abordar a mulher vitimizada que busca atendimento, mas silencia a respeito da violência sofrida, por vergonha ou medo.

A não identificação da violência nos serviços de saúde está atrelada à dificuldade dos profissionais de se envolverem em assuntos íntimos e complexos que envolvam situações de violência ${ }^{(13)}$. Nessa perspectiva, atenta-se para a capacitação dos profissionais da atenção primária de saúde frente a essas situações, dado que a intervenção sobre a violência ainda se mostra pouca efetiva.

Atos violentos contra a mulher são invisíveis no serviço e essa invisibilidade da violência contribui de forma significativa para práticas assistenciais fragilizadas. Dentre os principais motivos para a não contemplação da violência doméstica nas unidades de saúde, destaca-se: o sigilo das mulheres, o receio em intervir em assuntos conjugais, a não identificação das lesões físicas aparentes e a falta de tempo ${ }^{(22)}$.

Estudos $^{(11,23)}$ ressaltam que a violência contra a mulher se concretiza principalmente dentro do próprio domicílio, devido à privacidade e pouca interferência de outros indivíduos. Acrescenta-se também que, ao longo do tempo de união, a ocorrência de episódios violentos assume maior frequência; evidenciando, sobretudo, a violência psicológica, que é naturalizada e, por vezes, socialmente aceita ${ }^{(14,16,24)}$. Diante desse contexto, os resultados do presente estudo reforçam a importância dos profissionais de saúde conhecerem a realidade de vida das mulheres vitimadas que frequentam os serviços de saúde para melhor compreenderem e enfrentarem as situações de violência.

A construção de ações de enfrentamento da violência doméstica reúne particularidades consideráveis. A maior parte dos atos violentos não consiste em episódio único, mas uma série de ocorrências que podem chegar a perdurar por décadas, aumentando o risco para transtornos comuns, transtornos de estresse pós-traumático, síndrome da dor crônica, dificuldades de socialização e problemas na saúde reprodutiva, entre outras comorbidades ${ }^{(16)}$.

Entretanto, alerta-se que, quando os maus-tratos tendem a ocorrer nos primeiros anos de união e a mulher busca ajuda para a relação conflituosa, tal atitude favorece para que os agravos não se cronifiquem ${ }^{(14)}$. Nessa situação, é importante que a equipe de saúde esteja instrumentalizada para acolher a mulher para além das lesões físicas ou problemas orgânicos, incorporando em sua prática a abordagem em diferentes setores, como o de justiça, o de segurança pública, o de saúde e o de educação ${ }^{(16)}$.

$\mathrm{O}$ consumo de álcool pelo agressor é demonstrado em alguns estudos ${ }^{(15,25,26)} \operatorname{como}$ comportamento de risco associado à violência contra a mulher. As mulheres expressam ser o uso do álcool um fator deflagrante da violência conjugal. Tendo em vista que uma das características do agressor indicadas pelas vítimas no presente estudo é a predominância do uso do álcool e outras drogas, faz-se necessário que os serviços de atenção primária de saúde estejam preparados para incluir o agressor nas estratégias de enfrentamento da violência, oportunizando reestruturação e uma melhor coabitação familiar ${ }^{(25)}$.

O álcool, por ser aceito socialmente, tem largo consumo, principalmente entre os homens, tornando-o a droga mais nociva à dinâmica familiar ${ }^{(17,27)}$. As mulheres afirmam que a relação conjugal torna-se mais saudável quando se tem a suspensão de bebidas alcoólicas ${ }^{(25)}$. Reconhece-se que o álcool, como fator de risco modificável, necessita ser abordado na comunidade estudada com estratégias preventivas pela atenção primaria à saúde, visto que o seu uso exacerba a magnitude da violência, vulnerabilizando ainda mais as mulheres.

Conforme definições contidas na Carta de Otawa, as iniciativas de promoção da saúde representam esforço interdisciplinar e intersetorial de superar os vazios das abordagens tecnicistas e medicalizantes sobre os problemas de saúde ${ }^{(28)}$. O engajamento comunitário é, portanto, um componente crucial na medida em que favorece o mapeamento de suas reais necessidades e motivações, a colaboração entre múltiplos parceiros, e a responsabilização coletiva em torno dos resultados. 
A incorporação das práticas de promoção da saúde nos serviços da atenção primária de saúde propõe-se a articular toda a sociedade em prol da melhoria da qualidade de vida dos indivíduos. Esse processo é permeado por desafios, e as evidências de sucesso no campo da promoção da saúde podem orientar a sua replicação em diferentes cenários ${ }^{(29)}$.

Nesse contexto, a proposta de enfrentamento da violência contra as mulheres por meio de práticas de promoção da saúde e de estratificação da população com foco no âmbito local de atuação das unidades de saúde, conforme proposto pelo MACC, busca articular as ações da equipe da Estratégia Saúde da Família (ESF) com outros setores governamentais, não governamentais, movimentos sociais, igrejas, escolas e empresas, dentre outros ${ }^{(4,5)}$. As redes comunitárias de apoio são fundamentais para 0 incremento do capital e da coesão social, favorecendo a confiança, a solidariedade, a ausência de conflitos, a cultura da paz e a promoção da saúde. A intersetorialidade compreende as relações do setor saúde com os demais no intuito de desenvolver estratégias de atuação mais efetivas e sustentáveis, as quais não seriam obtidas isoladamente. Baseado nesse enfoque, o nível 1 do MACC propõe a execução de projetos que perpassem as intervenções de saúde e sejam capazes de promover melhorias nas condições gerais de vida por meio da geração de emprego para mulheres vitimizadas, do enfrentamento da drogadição, da organização de redes comunitárias de apoio e da adequada moradia ${ }^{(5)}$.

Com vistas a fortalecer as ações intersetoriais, o governo brasileiro propôs as diretrizes do Pacto Nacional pelo Enfrentamento à Violência contra a Mulher para implementação dos serviços em rede de identificação, de acolhimento e de atendimento integral das mulheres que vivenciam situações de violência, subdividindo-os em rede de enfrentamento e em rede de atendimento ${ }^{(3,30)}$.

A rede de enfrentamento refere-se à atuação entre as instituições governamentais, não governamentais e a comunidade, visando políticas de prevenção que garantam o empoderamento e a construção da autonomia das mulheres e dos seus direitos humanos. A rede de atendimento à mulher em situação de violência deve ser organizada entre diferentes setores, envolvendo, em especial, a assistência social, a justiça, a segurança pública e a saúde ${ }^{(3)}$.

A proposta de constituir redes de atenção parte da compreensão de que, embora a violência contra a mulher produza forte impacto sobre sua saúde, ela representa um problema mais amplo, de caráter social. Sua abordagem, portanto, requer recursos na área da saúde e em várias outras ${ }^{(19)}$. Apesar dos avanços para o enfrentamento da violência contra as mulheres, ainda se faz necessário o desenvolvimento de programas eficazes, com ações de prevenção e cuidado mais inclusivas e amplas ${ }^{(6)}$.

Os profissionais de saúde compreendem a rede de atenção como algo que perpassa o setor de saúde e da responsabilidade municipal. Visualizam a rede de forma positiva, como resultado da integração de áreas e de serviços; porém, a falta de articulação desses dificulta o seu funcionamento. No que diz respeito às estratégias realizadas na atenção primária de saúde, evidencia-se o acolhimento, as orientações, os encaminhamentos e a notificação ${ }^{(31)}$.

Dessa forma, o desenvolvimento de ações nos serviços de saúde capazes de enaltecer o processo saúde-doença no contexto social de vida das mulheres em situação de violência, bem como a escuta e a orientação qualificada entre profissional e usuário, colaboram para o fortalecimento de uma atenção integral alinhada à luta pelo empoderamento das mulheres ${ }^{(22)}$.

O nível 2 do MACC sugere intervenções de prevenção pautadas nas mudanças de comportamento modificáveis e de estilo de vida, estruturadas pela educação em saúde, pelo acesso à informação, pelo combate ao uso excessivo de álcool/drogas e pela criação de ambientes saudáveis ${ }^{(5)}$.

Para tanto, os fatores de riscos modificáveis precisam envolver metas no plano macroestrutural, com elaboração de legislação específica, por exemplo: aumento de imposto para as indústrias de álcool e de tabaco e restrição de venda de álcool no varejo. No plano mesoestrutural, deve englobar intervenções preventivas na comunidade, nas escolas e nos ambientes de trabalho. No plano microestrutural, ações educativas nas relações cotidianas entre os usuários e as equipes das unidades da $\mathrm{ESF}^{(5)}$

No entanto, mudança de comportamento é tarefa complexa e desafiadora. Com base em propostas do MACC, recomendase a utilização de modelos de abordagens ao usuário pelas equipes da ESF pautados em tecnologias leves, citando a entrevista motivacional, os grupos operativos e as intervenções educativas com base em evidências científicas ${ }^{(5)}$.

Posto isso, o MACC pode contribuir para assistência integral às vítimas e na minimização dos fatores prevalentes encontrados ao propor o uso de recursos comunitários a fim de melhorar a organização dos serviços de saúde e o uso de novas tecnologias de abordagem às condições de saúde ${ }^{(5)}$.

Dentre as limitações deste estudo, destaca-se a falta de registro de informações pelos profissionais de saúde, aspecto que interferiu na seleção das variáveis, mas que não inviabilizou a realização da pesquisa. Contudo, pondera-se que a ausência de padronização nas notificações contribui para a invisibilidade da violência perpetrada, uma vez que subestima a sua magnitude, gravidade, tipologia, localização de ocorrência e perfil das pessoas envolvidas.

\section{CONCLUSÃO}

A violência contra as mulheres obteve associação com a idade, a escolaridade e a renda da vítima, o uso abusivo de álcool pelo agressor e a relação conjugal, além de constatar-se que o conjunto de intervenções respaldadas pelo MACC para 
o enfrentamento da violência pode favorecer uma assistência integral às vítimas por englobar a complexidade dos fatores envolvidos, o autocuidado apoiado e a promoção de ambientes saudáveis.

\section{REFERÊNCIAS}

1. Brasil. Lei $\mathrm{n}^{\circ} 11340$ de 07 de agosto de 2006. Cria mecanismos para coibir a violência doméstica e familiar contra a mulher. Diário Oficial da União; Brasília; 8 ago. 2006.

2. Alexandre LBSP. Políticas públicas de saúde da mulher. In: Fernandes RAQ, Narchi NZ, organizadores. Enfermagem e saúde da mulher. $2^{\mathrm{a}}$ ed. Barueri: Manole; 2012. p. 7-27.

3. Presidência da República (BR), Secretaria de Políticas para as Mulheres. Rede de enfrentamento à violência contra as mulheres. Brasília: Secretaria de Políticas para as Mulheres; 2011 [acesso em 2017 Out 26]. Disponível em: https://www12. senado.leg.br/institucional/omv/entenda-a-violencia/pdfs/rede-de-enfrentamento-a-violencia-contra-as-mulheres

4. Mendes EV. As redes de atenção à saúde. Brasília: Organização Pan-Americana da Saúde; 2011 [acesso em 2017 Out 26 ]. Disponível em: http:/www.paho.org/bra/index.php?option=com_docman\&view=download\&category_slug=servicossaude-095\&alias=1402-as-redes-atencao-a-saude-2a-edicao-2\&Itemid $=965$

5. Mendes EV. O cuidado das condições crônicas na atenção primária à saúde: o imperativo da consolidação da estratégia da saúde da família. Brasília: Organização Pan-Americana da Saúde; 2012.

6. Barros EM, Silva MA, Falbo GH Neto, Lucena SG, Ponzo L, Pimentel AP. Prevalence and factors associated with intimate partner violence among women in Recife/Pernambuco, Brazil. Ciênc Saúde Colet. 2016;21(2):591-8.

7. Rodríguez-Borrego MA, Vaquero-Abellán M, Bertagnolli RL. Estudo transversal sobre fatores de risco de violência por parceiro íntimo entre enfermeiras. Rev Latinoam Enferm. 2012;20(1):11-8.

8. Gomes NP, Diniz NMF, Camargo CL, Silva MP. Homens e mulheres em vivência de violência conjugal: características socioeconômicas. Rev Gaúch Enferm. 2012;33(2):109-16.

9. Oliveira RNG, Fonseca RMGS. A violência como objeto de pesquisa e intervenção no campo da saúde: uma análise a partir da produção do Grupo de Pesquisa Gênero, Saúde e Enfermagem. Rev Esc Enferm USP. 2014; 48(Nesp 2):32-9.

10. Leite MTS, Figueiredo MFS, Dias OV, Vieira MA, Souza LPS, Mendes DC. Ocorrência de violência contra a mulher nos diferentes ciclos de vida. Rev Latinoam Enferm. 2014;22(1):85-92.

11. Andrade JO, Castro SS, Heitor SFD, Andrade WP, Atihe CC. Indicadores da violência contra a mulher provenientes das notificações dos serviços de saúde de Minas Gerais-Brasil. Rev Texto \& Contexto Enferm. 2016;25(3):1-9.

12. Acosta DF, Gomes VL, Barlem EL. Perfil das ocorrências policiais de violência contra a mulher. Rev Acta Paul Enferm. 2013;26(6):547-53.

13. Leite FMC, Amorim MHC, Wehrmeister FC, Gigante DP. Violência contra a mulher em Vitória, Espírito Santo, Brasil. Rev Saúde Pública. 2017;51:33.

14. Mathias AKRA, Bedone AJ, Osis MJD, Fernandes AMS. Prevalência da violência praticada por parceiro masculino entre mulheres usuárias da rede primária de saúde do Estado de São Paulo. Rev Bras Ginecol Obstet. 2013;35(4):185-91.

15. Vieira LJES, Ferreira RC, Moreira GAR, Gondim APS, Araujo MAL, Silva RM. Fatores associados à sobreposição de tipos de violência contra a mulher notificada em serviços sentinela. Rev Latinoam Enferm. 2013; 21(4):1-8.

16. Acosta FD, Gomes VLO, Fonseca AD, Gomes GC. Violência contra a mulher por parceiro íntimo: (in)visibilidade do problema. Texto \& Contexto Enferm. 2015; 24(1):121-7.

17. Silva CD, Gomes VLO, Mota MS, Gomes GC, Amarijo CL. Violence against women: aggressors drug users. Rev Pesqui Cuid Fundam [Internet]. 2015 [acesso em 2017 Out 26];7(2):2494-2504. Disponível em: http://bases.bireme.br/cgi-bin/ wxislind.exe/iah/online/?IsisScript $=$ iah/iah.xis\&src=google\&base=BDENF\&lang=p\&nextAction=lnk\&exprSearch=2683 8\&indexSearch=ID

18. Ilha MM, Leal SMC, Soares JSF. Mulheres internadas por agressão em um hospital de pronto socorro: (in)visibilidade da violência. Rev Gaúch Enferm. 2010;31(2):328-34.

19. Osis MJD, Duarte GA, Faúndes A. Violência entre usuárias de unidades de saúde: prevalência, perspectiva e conduta de gestores e profissionais. Rev Saúde Pública. 2012;46(2):351-8. 
20. Silva ACLG, Coelho EBS, Njaine K. Violência conjugal: as controvérsias no relato dos parceiros íntimos em inquéritos policiais. Ciênc Saúde Coletiva. 2014;19(4):1255-62.

21. García-Moreno C, Jansen HAFM, Elsberg M, Heise L, Watts CH. Prevalence of intimate partner violence: findings from the WHO multi-country study on women's health and domestic violence. Lancet. 2006;368(9543):1260-9.

22. Almeida LR, Silva ATMC, Machado LS. O objeto, a finalidade e os instrumentos do processo de trabalho em saúde na atenção à violência de gênero em um serviço de atenção básica. Interface Comum Saúde Educ. 2014;18(48):47-60.

23. Lucena KDT, Vianna RPT, Nascimento JA, Campos HFC, Oliveira ECT. Associação entre a violência doméstica e a qualidade de vida das mulheres. Rev Latinoam Enferm. 2017;25:e2901.

24. Lindner SR, Coelho EBS, Bolsoni CC, Rojas PF, Boing AF. Prevalência de violência física por parceiro íntimo em homens e mulheres de Florianópolis, Santa Catarina, Brasil: estudo de base populacional. Cad Saúde Pública. 2015;31(4):815-26.

25. Paixão GPN, Gomes NP, Diniz NMF, Couto TM, Vianna LAC, Santos SMP. Situações que precipitam conflitos na relação conjugal: o discurso de mulheres. Texto \& Contexto Enferm. 2014;23(4):1041-9.

26. Silva EB, Padoin SM, Vianna LA. Violência contra a mulher: limites e potencialidades da prática assistencial. Rev Acta Paul Enferm. 2013;26(6):608-13.

27. Mangueira SO, Lopes MVO. Família disfuncional no contexto do alcoolismo: análise de conceito. Rev Bras Enferm. 2014;67(1):149-54.

28. Magalhães R. Avaliação da Política Nacional de Promoção da Saúde: perspectivas e desafios. Ciênc Saúde Colet. 2016;21(6):1767-76.

29. Silva KL, Sena RR, Belga SMMF, Silva PM, Rodrigues AT. Promoção da saúde: desafios revelados em práticas exitosas. Rev Saúde Pública. 2014;48(1):76-85.

30. Acosta DF, Gomes VLO, Oliveira DC, Gomes GC, Fonseca AD. Aspectos éticos e legais no cuidado de enfermagem às vítimas de violência doméstica. Texto \& Contexto Enferm. 2017;26(3):e6770015.

31. Arboit J, Padoin SMM, Vieira LB, Paula CC, Costa MC, Cortes LF. Atenção à saúde de mulheres em situação de violência: desarticulação dos profissionais em rede. Rev Esc Enferm USP. 2017;51:e03207.

\section{Endereço para correspondência:}

Eliane Rolim de Holanda

Centro Acadêmico de Vitória

Rua Alto do Reservatório, s/n

Bairro: Bela Vista

CEP: 55608-680 - Vitória de Santo Antão - PE - Brasil

E-mail: elianerolim@yahoo.com.br 